\title{
SYNTHESIS AND STABILITY OF RESVERATROL-CONJUGATED GOLD NANOPARTICLES MODIFIED WITH POLYETHYLENE GLYCOL
}

\author{
RADITYA ISWANDANA, RICHA NURSELVIANA, SUTRIYO SUTRIYO*
}

Laboratory of Pharmaceutics and Pharmaceutical Technology, Faculty of Pharmacy, Universitas Indonesia, Depok 16424, Indonesia. Email: sutriyo@farmasi.ui.ac.id

Received: 02 October 2019, Revised and Accepted: 24 December 2019

\section{ABSTRACT}

Objective: Gold nanoparticles (AuNPs) are highly useful for drug delivery, but their application is limited by their stability as they readily aggregate. This issue can be prevented by adding a stabilizing agent such as resveratrol (RSV), which is a polyphenol derived from plants, that is used to prevent cancer. Therefore, we propose a novel method to prepare stable RSV-conjugated nanoparticles modified with polyethylene glycol (RSV-AuNP-PEG).

Methods: In the first step, the Turkevich method was used to synthesize the AuNPs. Then, PEG was added as stabilizer agent and conjugated with RSV. The synthesized conjugates were characterized using ultraviolet-visible spectrophotometry, Fourier transform infrared spectroscopy, particle size analysis, and high-performance liquid chromatography.

Results: The obtained RSV-AuNP-PEG had a particle size of $83.93 \mathrm{~nm}$ with a polydispersity index (PDI) of 0.562 and formed a translucent purple-red fluid in solution. The zeta potential was $-22.9 \mathrm{mV}$, and the highest entrapment efficiency was 75.86 $\pm 0.66 \%$. For comparison, the RSV-AuNP solution was purple and turbid, the particle size was $51.97 \mathrm{~nm}$ with a PDI of 0.694 , and the zeta potential was $-24.6 \mathrm{mV}$. The stability test results showed that the storage stability of RSV-AuNP-PEG was better than that of AuNP-RSV. Further, the RSV-AuNP-PEG was shown to be most stable in $2 \%$ bovine serum albumin (BSA) while the AuNP-RSV was most stable in 2\% BSA in phosphate-buffered saline pH 7.4.

Conclusion: These results show that modification of RSV-conjugated AuNPs with PEG effectively prevents their aggregation in storage, but only in certain mediums.

Keywords: Gold nanoparticles, Polyethylene glycol, Resveratrol, Stability.

(C) 2020 The Authors. Published by Innovare Academic Sciences Pvt Ltd. This is an open access article under the CC BY license (http://creativecommons. org/licenses/by/4. 0/) DOI: http://dx.doi.org/10.22159/ijap.2020.v12s1.FF050

\section{INTRODUCTION}

Nanoparticles have several advantages such as improvement in the intracellular infiltration, circulation time of the drug, and enhanced hydrophobic solubility [1]. Nanoparticles are usually $<100 \mathrm{~nm}$ in size. They consist of different biodegradable materials such as natural or synthetic polymers, lipids, or metals [2]. Gold nanoparticles (AuNPs) have been widely developed as a multifunctional platform for various biomedical applications [3] such as biosensing systems, cancer therapies, drug delivery, and pharmaceuticals [4]. AuNPs have core sizes ranging from 1 to $150 \mathrm{~nm}$. In general, AuNPs are synthesized by chemical reduction using chloroauric acid $\left(\mathrm{HAuCl}_{4}\right)$ as the reducing agent. AuNPs offer a combination of chemical, physical, optical, and electronic properties that are beneficial in various fields. However, AuNPs are unstable because of their high surface energy, which can cause them to aggregate. Aggregation can occur particularly in biological applications because of the high ionic strengths of many biological fluids and non-specific interactions between nanoparticles and biomolecules such as proteins or DNA [5]. This aggregation of AuNPs prevents their penetration into target cells. Particle aggregation can be prevented by adding a stabilizing agent [6] such as polyethylene glycol (PEG), which decreases the surface energy and Van der Waal's interactions, thereby reducing aggregation [7].

AuNPs can be conjugated with drugs such as resveratrol (RSV). RSV is a polyphenol compound found in plants, usually in its trans-RSV $\left(3,5,4^{\prime}\right.$-trihydroxystilbene) form [8], that has the ability to prevent cancer [9]. RSV can effectively inhibit the growth of breast cancer cells by inhibiting several molecules, including MMP-9, COX-2, NF$\alpha \mathrm{B}, \mathrm{AP}-1$, and PI3K/Akt dan ERK. This effect is stronger with AuNPs than with the same dose of RSV alone [10]. In this study, AuNPs were conjugated with RSV as a drug model and modified with PEG as a stabilizing agent. The obtained RSV-AuNPs-PEG were evaluated in terms of its physical stability and particle size and characterized by ultraviolet (UV)-visible spectrophotometry, Fourier transform infrared (FTIR) spectroscopy, and high-performance liquid chromatography (HPLC).

\section{MATERIALS AND METHODS}

\section{Materials}

RSV (Sigma-Aldrich, USA), ethanol (Merck, Germany), chloroauric acid (Sigma Aldrich, USA), PEG bisamine (molecular weight 3.000; Sigma-Aldrich, USA), sodium citrate (Merck, Germany), phosphatebuffered saline (PBS; Sigma-Aldrich, USA), cysteine (Sigma-Aldrich, USA), bovine serum albumin (BSA; Sigma-Aldrich, USA), and sodium hydroxide, methanol (Merck, Germany) were used. The solvents and other chemical reagents were of analytical grade.

\section{Synthesis of AuNP-RSV with PEG modification}

$15 \mathrm{ml}$ of a $1 \mathrm{mM}$ aqueous solution of $\mathrm{HAuCl}_{4} \cdot 3 \mathrm{H}_{2} \mathrm{O}$ was heated to $\pm 95^{\circ} \mathrm{C}$ while stirring. Then, $423 \mu \mathrm{l}$ of a $130 \mathrm{mM}$ sodium citrate aqueous solution was rapidly added to the solution while stirring. The solution changed from pale yellow to colorless, then changed to dark blue, and finally to deep red-burgundy after a few minutes. The solution was stirred and heated for $15 \mathrm{~min}$ after the addition of sodium citrate, then removed from heat and cooled at room temperature while stirring. $2.175 \mathrm{ml}$ of a $0.04 \mathrm{mM}$ aqueous PEG bisamine was added while stirring. Next, $3.425 \mathrm{ml}$ of aqueous RSV was added dropwise to the solution, and stirring was continued at $150 \mathrm{rpm}$ for $2 \mathrm{~h}$ at room temperature to obtain RSV-conjugated AuNPs-PEG (RSV-AuNPs-PEG). In addition, the RSV-AuNPs solution without PEG was also made (RSV-AuNPs). 


\section{Characterization}

Physical appearance

The physical appearance of RSV-AuNPs-PEG was observed, including the shape and color of the solution.

\section{Spectral analyses}

Absorbance and wavelength measurements were obtained with a UVvisible spectrophotometer over a wavelength range of 400-600 nm. The infrared spectrum was evaluated by FTIR spectroscopy over a wavenumber range of $400-4000 \mathrm{~cm}^{-1}$ on dried samples ground homogeneously with $\mathrm{KBr}$.

\section{Particle size distribution, polydispersity index (PDI), and zeta potential}

The particle size distributions and zeta potentials of RSV-AuNPs-PEG and RSV-AuNPs were measured by dynamic light scattering (DLS) using a particle size analyzer with a computerized system (Malvern, Zetasizer)

\section{Entrapment efficiency measurements}

$1.5 \mathrm{ml}$ of RSV-AuNP-PEG was ultracentrifuged at 12,000 rpm for $1 \mathrm{~h}$. The precipitate was discarded, and the supernatant was collected and filtered through a $0.22 \mu \mathrm{m}$-pore filter. The entrapment efficiency was measured by the indirect method using HPLC and calculated using the following formula:

$$
\mathrm{EE} \%=\frac{\text { Total RSV }- \text { Free RSV }}{\text { Total RSV }} \times 100 \%
$$

\section{Chromatography}

A C18 column was used to perform chromatography with the obtained samples. The mobile phase was Aqua Pro, which contains acetonitrile and glacial acetic acid. The injection volume was $20 \mu \mathrm{l}$, and the flow rate was $1.5 \mathrm{ml} / \mathrm{min}$. The eluted fractions were analyzed using a UV-visible $306 \mathrm{~nm}$ detector.

\section{Stability test in various mediums}

$1 \mathrm{ml}$ of each sample was re-dispersed in each $0.5 \mathrm{ml} \mathrm{PBS} \mathrm{pH} \mathrm{7.4,} \mathrm{PBS} \mathrm{pH} 4$, $0.9 \% \mathrm{NaCl}, 2 \% \mathrm{BSA}$, and $1 \%$ cysteine. The stability test was conducted over the course of 1 month. Samples were collected daily during the $1^{\text {st }}$ week and at 3 -day intervals during the 3 weeks. Each sample was evaluated in terms of the precipitates formed and absorbance using UVvisible spectrophotometry

\section{RESULTS AND DISCUSSION}

\section{Color changes}

RSV-AuNPs-PEG was synthesized with $1 \mathrm{mM} \mathrm{HAuCl}_{4} \cdot 3 \mathrm{H}_{2} \mathrm{O}$ and $130 \mathrm{nM}$ sodium citrate as the reducing agent. Sodium citrate reduced $\mathrm{Au}^{3+}$ to $\mathrm{Au}^{0}$ by reacting with $\mathrm{H}_{2} \mathrm{O}$ to form citric acid [11]. The reaction is as follows.

$2 \mathrm{HAuCl}_{4}+3 \mathrm{C}_{6} \mathrm{H}_{8} \mathrm{O}_{7}$ (citric acid) $\rightarrow 2 \mathrm{Au}+3 \mathrm{C}_{5} \mathrm{H}_{6} \mathrm{O}_{5}$ (3-acid ketoglutarate) $+8 \mathrm{HCl}+3 \mathrm{CO}$

The color change of the solution from pale yellow to colorless to dark blue then to deep red-burgundy or purple-reddish after a few minutes confirms the reduction from $\mathrm{Au}^{3+}$ to $\mathrm{Au}^{0}$ as the purple-reddish color arises from surface plasmon resonance, which is characteristic of metal nanoparticles. The reduction reaction can be further confirmed based on new absorbance in the wavelength range of 500-550 nm on the absorbance spectrum [12]. The absorbance spectra in this study showed increased absorption at $528.50 \mathrm{~nm}$, indicating that AuNPs were successfully formed (Fig. 1).

When $0.04 \mathrm{mM}$ PEG bisamine was added to the solution, the purplereddish was still formed, indicating that the nanoparticles remained stable when modified with PEG. PEG may reduce the attractive forces between particles and decrease the surface energy by steric stabilization, thereby preventing the aggregation that often occurs with AuNPs.

The RSV-AuNPs-PEG solution was also translucent with no odor, in contrast to the RSV-AuNPs solution without PEG. The lack of turbidity is characteristic of the colloidal dispersion of AuNPs and indicates that the RSV-AuNPs-PEG nanoparticles were small in size and homogeneously dispersed.

\section{Absorbance properties}

$\mathrm{HAuCl}_{4}$ exhibited strong absorbance at $287 \mathrm{~nm}$. This can be attributed to the charge transfer between metal and the chlorine-based ligand. When the $\mathrm{HAuCl}_{4}$ by the sodium citrate, the absorbance peak at $287 \mathrm{~nm}$ disappeared and a new absorbance peak formed at $529 \mathrm{~nm}$. As shown in Fig. 2, RSV-AuNPs-PEG and RSV-AuNPs absorbed light at the same wavelength, $529 \mathrm{~nm}$, but the shape of the spectra differed. RSV-AuNPPEG exhibited stronger absorbance than RSV-AuNPs, indicating that these nanoparticles were smaller [13]. RSV-AuNPs-PEG also had a relatively narrow absorption peak around $520 \mathrm{~nm}$, while RSV-AuNPs, with larger particles, had a wider absorption peak [14]; this means

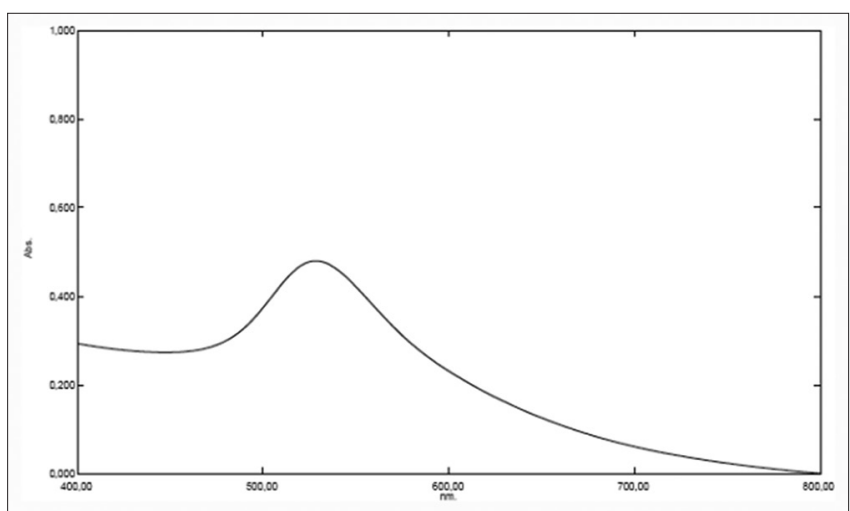

Fig. 1: Ultraviolet-visible spectra of gold nanoparticles, showing a peak at $528.50 \mathrm{~nm}$

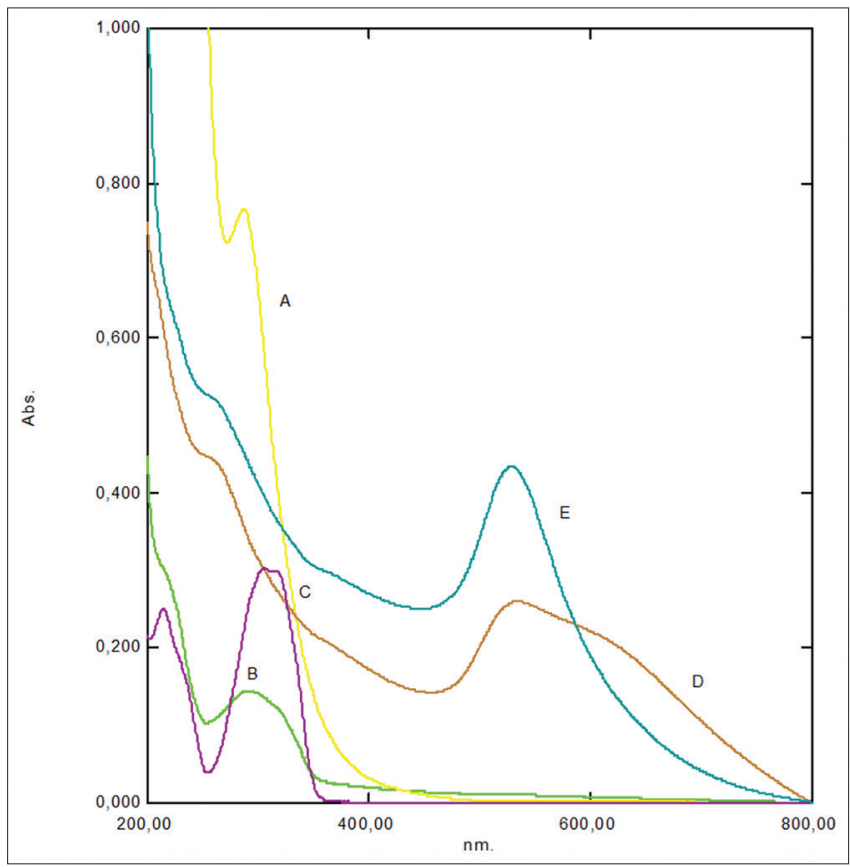

Fig. 2: Ultraviolet-visible absorbance spectra of (a) $1 \mathrm{mM} \mathrm{HAuCl}_{4}$,

(b) a 12 ppm polyethylene glycol (PEG) solution, (c) $10 \mathrm{ppm}$ resveratrol (RSV), (d) RSV-gold nanoparticles (AuNPs), (e) RSVAuNPs-PEG 
that the solution was monodisperse due to the stabilizing effect of PEG-bisamine, which prevented particle aggregation.

\section{Particle size distribution, PDI, and zeta potential}

The particle size, zeta potential, and PDI of each sample are shown in Table 1. DLS was used to determine the particle size; the measurement with this technique not only depends on the core size but also on the surface structure [15]. The particle size measurements show that the particles were nanoscale sized (1-100 nm) [16]: RSV-AuNPs-PEG had a particle size of $83.93 \mathrm{~nm}$ while RSV-AuNPs had a particle size of $51.97 \mathrm{~nm}$. These results confirm that the RSV-AuNPs-PEG particles were larger than the RSV-AuNPs particles. This difference can be attributed to the PEG caps on the RSV-conjugated AuNPs.

Another important physical property is the PDI, which reflects the degree of heterogeneity of particles. The particle size distribution was measured using a DLS particle size analyzer (Malvern Zetasizer). PDIs closer to zero imply that the particle size is more homogeneous; PDIs $<0.7$ are considered to be good [17]. The PDI of RSV-AuNPs was 0.694 and that of RSV-AuNPs-PEG was 0.562 .

The zeta potentials of RSV-AuNPs and RSV-AuNPs-PEG were measured. Zeta potentials $>+30 \mathrm{mV}$ or $<-30 \mathrm{mV}$ are considered to be good [13].

Table 1: Size, PDI, and zeta potential of each sample

\begin{tabular}{llll}
\hline Formula & Size $(\mathbf{n m})$ & PDI & Zeta potential (mV) \\
\hline RSV-AuNPs & 51.97 & 0.694 & -22.9 \\
RSV-AuNPs-PEG & 83.93 & 0.562 & -24.6 \\
\hline
\end{tabular}

PDI: Polydispersity index, RSV: Resveratrol, AuNPs: Gold nanoparticles, PEG: Polyethylene glycol
The zeta potential of RSV-AuNPs was $-24.6 \mathrm{mV}$ and that of RSV-AuNPsPEG was $-22.9 \mathrm{mV}$. While both zeta potential values were $>-30 \mathrm{mV}$, that of the RSV-AuNPs-PEG was larger than that of the RSV-AuNPs. This is because the PEG modification decreases the zeta potential [18], which may be because PEG leads to steric stabilization that does not significantly affect the electrokinetic potential in the colloidal dispersion.

\section{Infrared spectra}

The FTIR spectra of AuNPs, RSV, RSV-AuNPs, and RSV-AuNPs-PEG are shown in Fig. 3. RSV exhibited prominent bands at wavenumbers of 3380-2925 and 2834-2456 (attributed to 0-H), 1613 (attributed to $\mathrm{C}=\mathrm{C}$ alkene), 1600, 1475 ( $\mathrm{C}=\mathrm{C}$ aromatic), and $833 \mathrm{~cm}^{-1}$ (meta-para substitution). Bands were observed at 833 (meta-para substitution), 1475 , and $1600 \mathrm{~cm}^{-1}$ ( $\mathrm{C}=\mathrm{C}$ aromatic) in RSV-AuNPs and RSV-AuNPsPEG, implying that there may be RSV in samples. The PEG-bisamine exhibited prominent bands at wavenumbers of $3621(\mathrm{~N}-\mathrm{H}), 2864$ $(\mathrm{C}-\mathrm{H}), 1298(\mathrm{C}-\mathrm{O}), 1131(\mathrm{C}-\mathrm{N})$, and $1600 \mathrm{~cm}^{-1}(\mathrm{C}=\mathrm{C})$. However, the bands at $1600\left(\mathrm{C}=\mathrm{C}\right.$ alkene), $1100(\mathrm{C}-\mathrm{N})$, and $3621 \mathrm{~cm}^{-1}(\mathrm{~N}-\mathrm{H})$ were not observed in the spectrum of RSV-AuNPs-PEG. Therefore, it was suspected there a bond had formed with the N group due to the PEGbisamine.

\section{Entrapment efficiency}

The standard calibration data for the entrapment efficiency (Fig. 4) were fitted to a curve with the equation of $y=92620 x-28703$ ( $y=$ peak area; $\mathrm{x}=$ concentration) with $\mathrm{r}=0.9995$. The chromatogram showed time retentions of both standard RSV and the RSV in the sample were about 14 min (Figs. 4 and 5). The entrapment efficiency analysis with an indirect method showed RSV concentration that entrapment in RSVAuNPs-PEG in 3 times analysis was 76.59\%, 75.29\%, and 75.70\%. The entrapment efficiency in this sample was $75.86 \pm 0.6 \%$

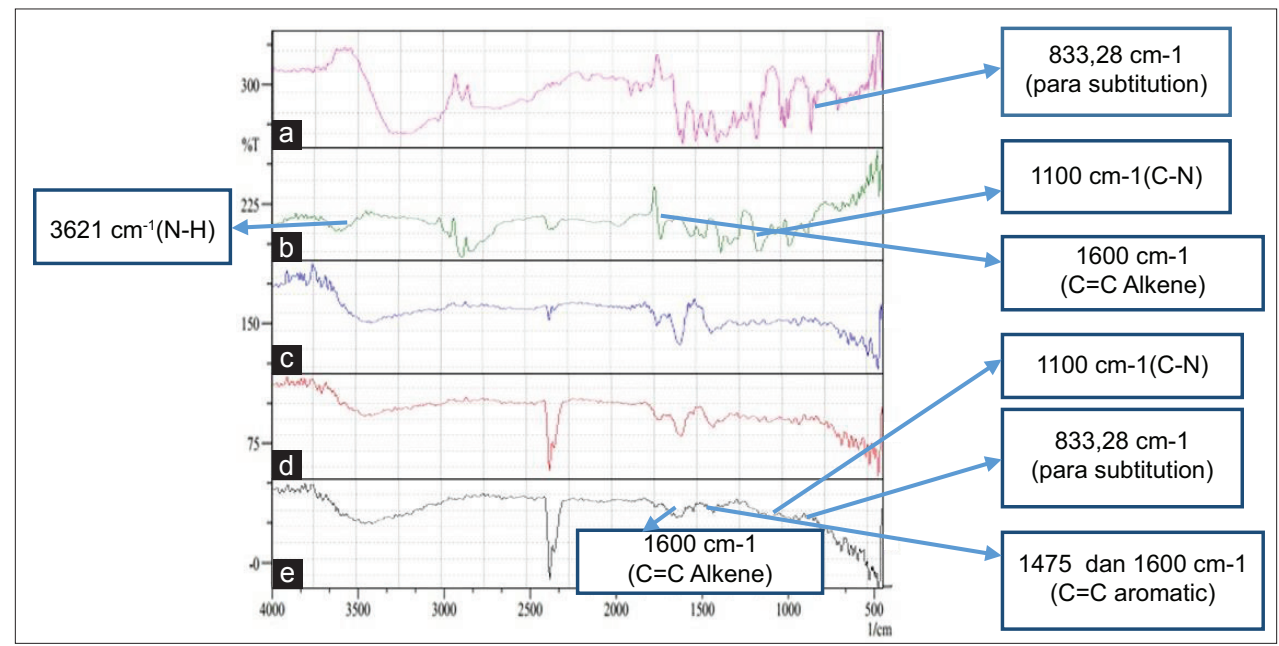

Fig. 3: Infrared spectra of (a) resveratrol (RSV), (b) polyethylene glycol (PEG)-bisamine, (c) gold nanoparticles (AuNPs), (d) AuNPs-RSV, and (e) RSV-AuNPs-PEG

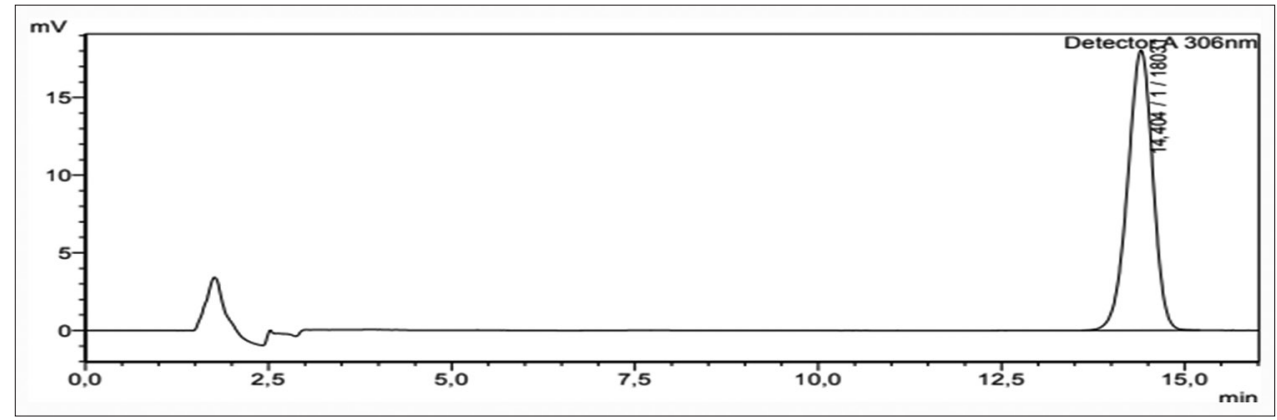

Fig. 4: Chromatogram of the resveratrol standard 


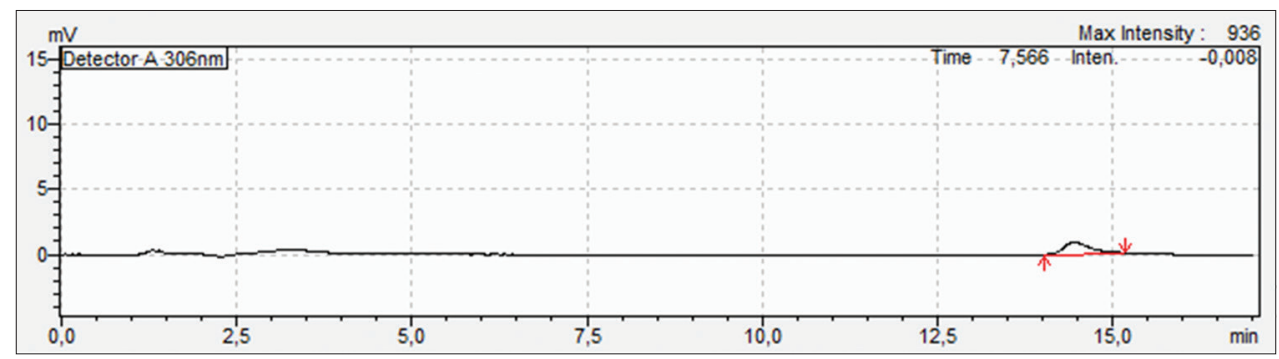

Fig. 5: Chromatogram of resveratrol in the sample

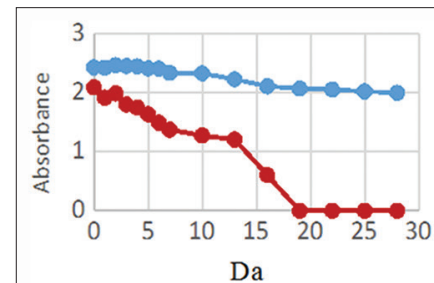

a $\longrightarrow$ Absrobance of RSV-AunP.PE

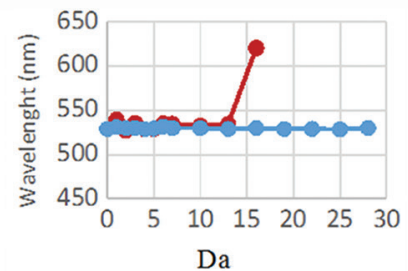

$\mathrm{Da}$

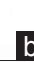

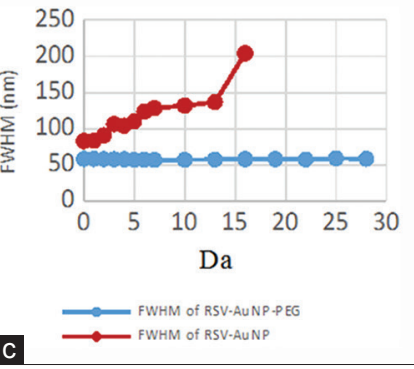

C $\longrightarrow$ FWHM of RSV-AUNP

Fig. 6: Comparisons of the (a) absorbance, (b) wavelength, and (c) full width at half maximum for resveratrol-gold nanoparticles (RSVAuNPs)-polyethylene glycol and RSV-AuNPs over 1 month

\section{Stability in various media}

Stability tests were conducted to evaluate the possibility of aggregated AuNPs in the body, which is important because unstable AuNPs would be dangerous for the body. Another purpose of these measurements was to evaluate which media can distribute the AuNPs. Samples were stored for 4 weeks in different mediums then evaluated using a UV-visible spectrophotometer as absorbance decreases indicate larger particle sizes [13]. The physical appearance was observed, and the absorbance changes were evaluated in terms of the maximum wavelength shift and full width at half maximum (FWHM). The absence of changes in the absorbance and wavelength of the UV-visible spectrum indicates that the AuNPs were stable [14]. A low FWHM indicates monodispersity while a high FWHM indicates polydispersity [19]; thus, the wider the FWHM is, the more unequal the sizes of the colloidal AuNPs, which may indicate that the AuNPs aggregated. The results are shown in Fig. 6 and summarized in Table 2 .

\section{Stability of control (without addition medium)}

The control sample was sampled without redispersing at any of the media. The UV-visible absorbance and wavelength of the RSV-AuNPsPEG solution decreased between days 0 and 28, but the change was not significant. On the other hand, those of RSV-AuNPs fluctuated over the 4 weeks. This result demonstrates that RSV-AuNPs-PEG was more stable than RSV-AuNPs. In addition, the shape of the absorbance spectrum of RSV-AuNPs-PEG was different from that of RSV-AuNPs in that it was relatively narrow. This difference was confirmed by the observation that the FWHM of RSV-AuNPs-PEG did not increase significantly over the experimental period, while that of RSV-AuNPs increased at each sampling time point. Furthermore, the absorbance bands of RSV-AuNPs shifted to longer wavelengths [20] compared with those of RSV-AuNPsPEG, indicating that it had a larger particle size. This finding implies that the RSV-AuNPs may have aggregated. This was supported by the observed precipitate formation on day 16. Based on these findings, it can be concluded that RSV-AuNPs-PEG was more stable in storage than RSV-AuNPs.

\section{Stability in $1 \%$ cysteine}

The addition of cysteine to the RSV-AuNPs-PEG solution caused an instant color change from purple-reddish to blue, which indicates that the solution was not stable in this medium. The UV-visible absorbance spectrum of this sample shows widening, indicating that the AuNPs had coagulated in this medium [14]. In addition, the spectrum also showed new absorbance at $680 \mathrm{~nm}$, which occurs due to oscillating plasma dipoles when two metal particles of a colloid join and their plasma dipoles interact [20]. Similar observations were made with the RSVAuNPs, indicating that it was also unstable in the $1 \%$ cysteine medium. This instability can be attributed to the interactions between the AuNPs and the thiol groups in cysteine, which are stronger than the bonds between the PEG-bisamine and cause the AuNPs to aggregate.

\section{Stability in $0.9 \% \mathrm{NaCl}$}

The color of the RSV-AuNPs solution changed from reddish-purple to purple in the $0.9 \% \mathrm{NaCl}$ medium, while that of the RSV-AuNPs-PEG solution changed from reddish-purple to blue, reflecting the instability of both samples in this medium. On day 1, both solutions became clear, and a precipitate was observed. In addition, at this time point, the absorbances of the AuNPs-RSV and RSV-AuNPs-PEG solutions decrease significantly, further demonstrating their instability. Comparing the FWHM values in the $\mathrm{NaCl}$ medium, the spectra of both solutions were wide on day 0 . On the $1^{\text {st }}$ day, the FWHM value could not be calculated because the line was almost 0 due to the instability in this medium. The observed instability can be attributed to the $\mathrm{Na}^{+}$and $\mathrm{Cl}^{-}$ions causing the AuNPs to aggregate and precipitate according to the DLVO theory, which states that the coagulation of dispersed particles is explained in two forces: Van der Waals forces and repulsive electrostatic doublelayer forces [20].

\section{Stability in 2\% BSA}

A stability test was conducted in a BSA medium because albumin is the most abundant protein in the body, accounting for $60 \%$ of the protein in human plasma. Therefore, the stability test in BSA can predict the stability of drug-conjugated AuNPs in human blood plasma in vitro [21]. The results show that RSV-AuNPs-PEG was initially stable, but the absorbance of the RSV-AuNPs-PEG solution decreased and shifted from $525-526 \mathrm{~nm}$ to $523 \mathrm{~nm}$ on day 5 . This finding was supported by the formation of a precipitate in the RSV-AuNPs-PEG solution on day 5. In contrast, the absorption of the RSV-AuNPs solution was more stable in the BSA. Furthermore, the FWHM of the RSV-AuNPs-PEG solution was greater on day 5 than on day 0 , while that of the RSV-AuNPs solution did not change significantly, further confirming that RSV-AuNPs was more stable than RSV-AuNPs-PEG in this medium. The instability of RSV-AuNPs-PEG in this medium likely arose because PEG, which acts as a stabilizing agent, can also bind to proteins, leaving the AuNPs unstable leading to their aggregation, resulting in decreased and 
Table 2: RSV-AuNPs and RSV-AuNPs-PEG stabilities in various mediums

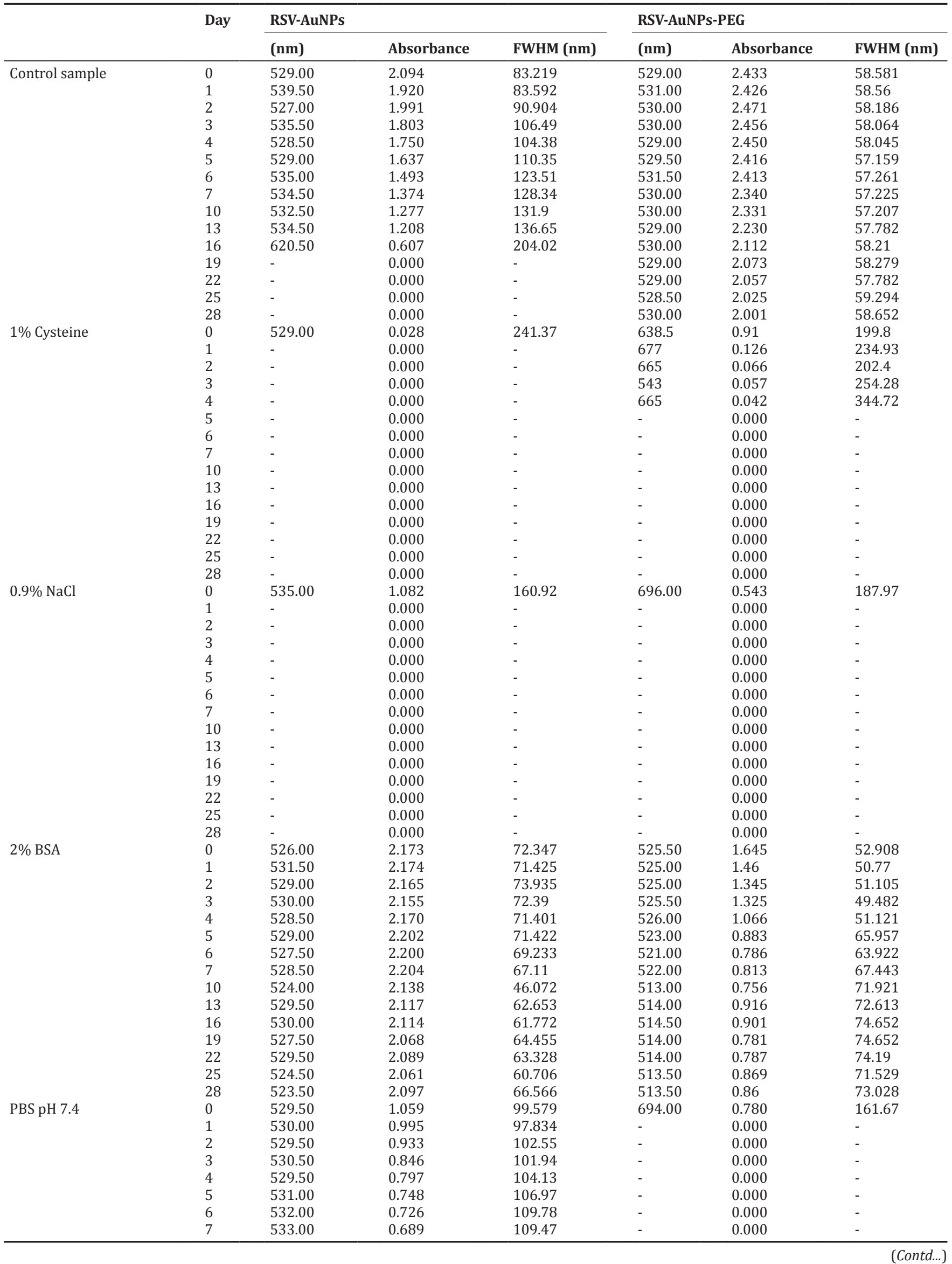


Table 2: (Continued)

\begin{tabular}{|c|c|c|c|c|c|c|c|}
\hline & \multirow[t]{2}{*}{ Day } & \multicolumn{3}{|c|}{ RSV-AuNPs } & \multicolumn{3}{|c|}{ RSV-AuNPs-PEG } \\
\hline & & $(\mathrm{nm})$ & Absorbance & FWHM (nm) & (nm) & Absorbance & FWHM (nm) \\
\hline & 10 & 533.50 & 0.654 & 111.3 & - & 0.000 & - \\
\hline & 13 & 533.00 & 0.629 & 108.14 & - & 0.000 & - \\
\hline & 16 & 532.50 & 0.618 & 109.8 & - & 0.000 & - \\
\hline & 19 & 532.50 & 0.590 & 112.14 & - & 0.000 & - \\
\hline & 22 & 532.00 & 0.544 & 135.84 & - & 0.000 & - \\
\hline & 25 & 532.50 & 0.580 & 153.31 & - & 0.000 & - \\
\hline & 28 & 530.50 & 0.582 & 150.15 & - & 0.000 & - \\
\hline \multirow[t]{15}{*}{ PBS pH 7.4} & 0 & 529.00 & 0.014 & 212.27 & 691 & 0.982 & 167.25 \\
\hline & 1 & - & 0.000 & - & 682 & 0.009 & 222.37 \\
\hline & 2 & - & 0.000 & - & - & - & - \\
\hline & 3 & - & 0.000 & - & - & - & - \\
\hline & 4 & - & 0.000 & - & - & - & - \\
\hline & 5 & - & 0.000 & - & - & - & - \\
\hline & 6 & - & 0.000 & - & - & - & - \\
\hline & 7 & - & 0.000 & - & - & - & - \\
\hline & 10 & - & 0.000 & - & - & - & - \\
\hline & 13 & - & 0.000 & - & - & - & - \\
\hline & 16 & - & 0.000 & - & - & - & - \\
\hline & 19 & - & 0.000 & - & - & - & - \\
\hline & 22 & - & 0.000 & - & - & - & - \\
\hline & 25 & - & 0.000 & - & - & - & - \\
\hline & 28 & - & 0.000 & - & - & - & - \\
\hline
\end{tabular}

RSV: Resveratrol, AuNPs: Gold nanoparticles, PEG: Polyethylene glycol, FWHM: Full width at half maximum, BSA: Bovine serum albumin, PBS: Phosphate-buffered saline

shifted absorbance [21]. We used PEG 3000 in this study; thus it has been considered as a long-chain PEG. In a previous study, long-chain PEG in aqueous solutions is more capable of interacting with proteins than short-chain PEG [21] because it contains more binding sites. Hence, it can be concluded that RSV-AuNPs is stable for 28 days in $2 \%$ BSA medium, but RSV-AuNPs-PEG became unstable on day 5 in this medium. This finding implies that PEG-modified particles are not suitable for in vitro applications.

\section{Stability in PBS}

Stability testing was conducted in PBS mediums of different pHs to determine the effect of $\mathrm{pH}$ on the AuNPs stability. $\mathrm{pH}$ change is one of the causes of aggregation in colloidal AuNPs solutions [20]. In this test, stability testing was performed at $\mathrm{pH} 7.4$, which represents the $\mathrm{pH}$ of the body under normal circumstances, and $\mathrm{pH} 4$, which mimics the environment of tumor tissue [22]. In the PBS pH 7.4 medium, RSVAuNPs was more stable than RSV-AuNPs-PEG, which indicates that the $\mathrm{pH}$ of the medium caused the dielectric modification of the RSV-AuNPsPEG, resulting in their aggregation. In contrast, the absorbance and wavelength of RSV-AuNPs did not decrease as significantly. Thus, the behavior of RSV-AuNPs-PEG may be derived from PEG-bisamine, which reacts with PBS. On the other hand, in the PBS pH four medium, both the RSV-AuNPs-PEG and RSV-AuNPs were unstable. Therefore, it can be presumed that the RSV will be readily released from nanoparticles in a tumor environment. The stability test results showed that RSV-AuNPsPEG was more stable over 28 days, while RSV-AuNPs was only stable for 19 days of storage. This difference can be attributed to the steric stabilization effects of PEG on the colloidal AuNPs.

\section{CONCLUSION}

The results of this study show that PEG modification of RSV-conjugated AuNPs prevents their aggregation in storage. However, this influence is only effective in some mediums. Although RSV-AuNPs-PEG was stable in BSA for 4 days, it was unstable in $1 \%$ cysteine, $2 \%$ BSA, $0.9 \%$ $\mathrm{NaCl}$, and PBS $\mathrm{pH}$ 7.4. On the other hand, RSV-AuNPs were stable in BSA $2 \%$ and PBS pH 7.4 for 28 days but unstable on 1\% cysteine, $0.9 \%$ $\mathrm{NaCl}$, and PBS pH 4. RSV-AuNPs were more stable most mediums than RSV-AuNPs-PEG. This difference may have been due to the low PEG concentration used, short stirring time such that the citrate ions were not fully exchanged with PEG, or the effect of temperature when the
PEG was added. Therefore, additional studies should be undertaken to optimize other parameters, such as the stirring time, temperature, and $\mathrm{pH}$, which affect the formation of the RSV-AuNPs-PEG conjugate. In addition, increasing the concentration of PEG in the synthesis process may improve its stabilizing effects.

\section{ACKNOWLEDGMENT}

We gratefully acknowledge the funding from Directorate of Research and Community Engagement Universitas Indonesia, via Hibah PITTA 2018 supporting this work.

\section{CONFLICTS OF INTEREST}

The authors declare that there are no conflicts of interest.

\section{REFERENCES}

1. Kadian R. Nanoparticles: A promising drug delivery approach. Asian J Pharm Clin Res 2018;11:30-5.

2. Lavanya N, Muzib I, Jithan A, Umamahesh B. Novel nanoparticles for the oral delivery of low molecular weight heparin: In vitro and in vivo assessment. Asian J Pharm Clin Res 2017;10:254-61.

3. Rahme K, Chen L, Hobbs R, Morris M, O'Driscoll C, Holmes J. PEGylated gold nanoparticles: Polymer quantification as a function of PEG lengths and nanoparticle dimensions. RSC Adv 2013;3:6085-94.

4. Ajnai G, Chiu A, Kan T, Cheng C, Tsai T, Chang J. Trends of gold nanoparticle-based drug delivery system in cancer therapy. J Exp Clin Med 2014;6:172-8.

5. Rahme K, Nolan M, Doody T, McGlacken G, Morris M, O'Driscoll C, et al. Highly stable PEGylated gold nanoparticles in water: Applications in biology and catalysis. RSC Adv 2013;3:21016-24.

6. Djajadisastra J, Sutiyo S, Purnamasari P, Pujiyanto A. Antioxidant activity of gold nanoparticles using gum Arabic as a stabilizing agent. Int J Pharm Pharm Sci 2014;6:462-5.

7. Kalaivani A, Chellaram C. Nanoparticle PEGylation for imaging and therapy- a review. Indian J Innov Dev 2012;1:52-3.

8. Kasiotis KM, Pratsinis H, Kletsas D, Haroutounian SA. Resveratrol and related stilbenes: Their anti-aging and anti-angiogenic properties. Food Chem Toxicol 2013;61:112-20.

9. Urbaniak A, Delgado M, Kacprzak K, Chambers TC. Activity of resveratrol triesters against primary acute lymphoblastic leukemia cells. Bioorg Med Chem Lett 2017;27:2766-70

10. Park SY, Chae SY, Park JO, Lee KJ, Park G. Gold-conjugated resveratrol nanoparticles attenuate the invasion and MMP-9 and COX- 
2 expression in breast cancer cells. Oncol Rep 2016;35:3248-56.

11. Kumar S, Gandhi KS, Kumar R. Modeling of formation of gold nanoparticles by citrate method. Ind Eng Chem Res 2007;46:3128-36.

12. Dyah PR, Djajadisastra J, Mutalib A. Pujiyanto A. Preparation of gold nanoparticles with based on conjugated gum Arabic vincristine and evaluation of their in vitro characteristics. J Ilmu Kefarmasian Indones 2018; $16: 6-11$

13. Chen KS, Hung TS, Wu HM, Wu JY, Lin MT, Feng CK. Preparation of thermosensitive gold nanoparticles by plasma pretreatment and UV grafted polymerization. Thin Solid Films 2010;518:7557-62.

14. Turkevich J. Colloidal gold (part II): Colour, coagulation, adhesion, alloying and catalytic properties. Gold Bull 1985;18:125-31.

15. Malvern Instrument. Dynamic Light Scattering: An Introduction in 30 Minutes. United Kingdom: Malvern Instrument; 2012.

16. Bhatia S. Natural Polymer Drug Delivery Systems: Nanoparticles Types, Classification, Characterization, Fabrication Methods, and Drug Delivery Applications. Switzerland: Springer; 2016

17. Ramadon D, Wirarti G, Anwar E. Novel transdermal ethosomal gel containing green tea (Camellia sinensis L. Kuntze) Leaves extract: Formulation and in vitro penetration study. J Young Pharm 2017;9:336-40.

18. Hou Z, Zhan C, Jiang Q, Hu Q, Li L, Chang D, et al. Both FA- and mPEG-conjugated chitosan nanoparticles for targeted cellular uptake and enhanced tumor tissue distribution. Nanoscale Res Lett 2011;6:563.

19. Oliveira J, Prado A, Keijok W, Ribeiro M, Pontes M, Nogueira B, et al. A helpful method for controlled synthesis of monodisperse gold nanoparticles through response surface modeling. Arab J Chem 2017; $13: 1-11$

20. Butt H, Graf K, Kapp M. Physics and Chemistry of Interfaces. Weinheim: Wiley-VCH; 2006

21. Wu J, Zhao C, Lin W, Hu R, Wang Q, Chen H, et al. Binding characteristics between polyethylene glycol (PEG) and proteins in aqueous solution. J Mater Chem B 2014;2:2983.

22. Swietach P, Vaughan-Jones RD, Harris AL, Hulikova A. The chemistry, physiology and pathology of $\mathrm{pH}$ in cancer. Philos Trans R Soc Lond B Biol Sci 2014:369:20130099. 